\title{
Electroencephalographic monitoring in comatose survivors of cardiac arrest
}

\author{
Stéphane Legriel \\ See related research by Alvarez et al., http://ccforum.com/content/17/5/R190
}

\begin{abstract}
Electroencephalography (EEG) monitoring is an important tool in the management of comatose survivors of cardiac arrest. The results serve to predict the neurological outcome, identify postanoxic status epilepticus, and assess the effectiveness of antiepileptic treatments. Continuous EEG monitoring might seem the most attractive option but is costly and requires the continuous availability of an expert to interpret the findings. Alvarez and colleagues compared repeated standard EEG to continuous EEG monitoring in comatose survivors of cardiac arrest. They found close agreement between these two strategies. However, their results do not constitute evidence of similar performance. In comatose survivors of cardiac arrest, repeated standard EEG should be used only when continuous EEG monitoring is unavailable.
\end{abstract}

In an interesting study in 34 comatose survivors of cardiac arrest (CSCAs), Alvarez and colleagues [1] compared the performance of repeated standard electroencephalography (sEEG) and continuous EEG (cEEG). They evaluated the two main objectives of EEG monitoring in CSCAs, namely the assessment of background activity and reactivity and the detection of postanoxic status epilepticus (PSE).

All 34 patients underwent cEEG. The entire cEEG recording was compared to two extracts, which served to replicate sEEG. The extracts were taken early during therapeutic hypothermia (TH) and shortly after the return to normothermia, respectively. Thus, each patient served as his or her own control. Agreement between sEEG and cEEG was $97.1 \%$ (95\% confidence interval, 84.6 to $99.9 \%$ ) for evaluating background discontinuity

Correspondence: slegriel@ch-versailles.fr

Intensive Care Unit, Centre Hospitalier de Versailles - Site André Mignot, 177 rue de Versailles, 78150, Le Chesnay cedex, France and reactivity and $94.1 \%$ (95\% confidence interval, 80.3 to $99.2 \%$ ) for PSE detection.

EEG has always had a major role in the management of CSCAs. Studies done after the introduction of TH confirmed the crucial contribution of EEG to neurological monitoring. Cerebral background activity and reactivity in the absence of sedation are among the most reliable predictors of the neurological outcome [2-5]. In addition, EEG monitoring ensures the detection of PSE during $\mathrm{TH}$, at a time when neuromuscular blocker therapy eliminates any clinical seizures [6]. EEG monitoring also provides information on the effectiveness of the treatment given to control PSE [7,8]. Finally, after rewarming and neuromuscular blocker withdrawal, EEG monitoring helps to characterize subcortical postanoxic myoclonus, which must be distinguished from PSE [9].

As emphasized by Alvarez and colleagues, EEG monitoring can be carried out continuously or intermittently. Despite the absence of proof of superiority, cEEG was recommended recently for the management of CSCAs $[2,10]$. However, the equipment and staff time needed for cEEG monitoring are not available in all ICUs. In addition, cEEG is of interest only if an expert is continuously available for real-time interpretation of the traces. The study by Alvarez and colleagues is therefore important. It sheds light on the performance of cEEG versus sEEG in CSCAs.

However, caution is in order regarding the practical impact of the results obtained by Alvarez and colleagues. In several studies that used continuous amplitudeintegrated EEG or cEEG, a continuous background pattern during $\mathrm{TH}[5,11,12]$ or normothermia $[5,13]$ was almost invariably associated with recovery. Similarly, reactive cEEG background activity was associated with recovery, whereas nonreactive cEEG background activity during $\mathrm{TH}$ predicted death [8]. In the study by Alvarez and colleagues, early sEEG would have led to an erroneous assessment of the potential for recovery in 1 of the 34 patients, whose background activity and reactivity 
improved a few hours after the cardiac arrest. Such changes in background activity and reactivity would probably be more common with sedation protocols using propofol instead of midazolam (as used in the study by Alvarez and colleagues).

Second, seizure activity or PSE is identified in up to $40 \%$ of CSCAs [14]. PSE occurs during TH in about $15 \%$ of patients [6], during rewarming in $8 \%$ to $20 \%[5,11]$, and shortly after the return to normothermia in $33 \%$ [11]. Whereas PSE is independently associated with poor outcomes, favourable outcomes have been reported in some patients with PSE occurring after (but not during) $\mathrm{TH}[6,7,14,15]$. Early identification and aggressive treatment of PSE may improve outcomes, although this possibility remains unproven to date. Nevertheless, early PSE diagnosis and treatment are currently a major objective in CSCAs. According to the study by Alvarez and colleagues, obtaining two sEEG recordings, during TH and after the return to normothermia, respectively, would miss $25 \%$ of all cases of PSE [1]. Moreover, the rewarming phase carries a high risk of PSE but was not investigated in this study.

Alvarez and colleagues have produced useful information on EEG monitoring strategies in CSCAs. However, their results do not indicate that sEEG performs as well as cEEG. In CSCAs, sEEG should be used only when cEEG is unavailable.

\footnotetext{
Abbreviations

CEEG: Continuous electroencephalography; CSCA: Comatose survivor of cardiac arrest; EEG: Electroencephalography; PSE: Postanoxic status epilepticus; sEEG: Standard electroencephalography; TH: Therapeutic hypothermia.
}

\section{Competing interests}

The author declares that they have no competing interests.

Published: 11 Nov 2013

\section{References}

1. Alvarez V, Sierra-Marcos A, Oddo M, Rossetti AO: Yield of intermittent versus continuous EEG in comatose survivors of cardiac arrest treated with hypothermia. Crit Care 2013, 17:R190.

2. Sutter R, Stevens RD, Kaplan PW: Continuous electroencephalographic monitoring in critically ill patients: indications, limitations, and strategies. Crit Care Med 2013, 41:1124-1132.

3. Rossetti AO, Oddo M, Logroscino G, Kaplan PW: Prognostication after cardiac arrest and hypothermia: a prospective study. Ann Neurol 2010, 67:301-307

4. Fugate JE, Wijdicks EF, Mandrekar J, Claassen DO, Manno EM, White RD, Bell MR, Rabinstein AA: Predictors of neurologic outcome in hypothermia after cardiac arrest. Ann Neurol 2010, 68:907-914.

5. Crepeau AZ, Rabinstein AA, Fugate JE, Mandrekar J, Wijdicks EF, White RD, Britton JW: Continuous EEG in therapeutic hypothermia after cardiac arrest: prognostic and clinical value. Neurology 2013, 80:339-344.

6. Legriel S, Bruneel F, Sediri H, Hilly J, Abbosh N, Lagarrigue MH, Troche G, Guezennec P, Pico F, Bedos JP: Early EEG monitoring for detecting postanoxic status epilepticus during therapeutic hypothermia: a pilot study. Neurocrit Care 2009, 11:338-344.

7. Rossetti AO, Logroscino G, Liaudet L, Ruffieux C, Ribordy V, Schaller MD, Despland PA, Oddo M: Status epilepticus: an independent outcome predictor after cerebral anoxia. Neurology 2007, 69:255-260.
8. Rossetti AO, Urbano LA, Delodder F, Kaplan PW, Oddo M: Prognostic value of continuous EEG monitoring during therapeutic hypothermia after cardiac arrest. Crit Care 2010, 14:R173.

9. Legriel S, Le Stang MB, Merceron S, Cronier P, Troche G: Ongoing abdominal status myoclonus in postanoxic coma. Neurocrit Care 2011, 16:136-138.

10. Claassen J, Taccone FS, Horn P, Holtkamp M, Stocchetti N, Oddo M: Recommendations on the use of EEG monitoring in critically ill patients: consensus statement from the neurointensive care section of the ESICM. Intensive Care Med 2013, 39:1337-1351.

11. Rundgren $M$, Westhall $E$, Cronberg T, Rosen I, Friberg H: Continuous amplitude-integrated electroencephalogram predicts outcome in hypothermia-treated cardiac arrest patients. Crit Care Med 2010, 38:18381844.

12. Cloostermans MC, van Meulen FB, Eertman CJ, Hom HW, van Putten MJ: Continuous electroencephalography monitoring for early prediction of neurological outcome in postanoxic patients after cardiac arrest: a prospective cohort study. Crit Care Med 2012, 40:2867-2875.

13. Rundgren $M$, Rosen I, Friberg H: Amplitude-integrated EEG (aEEG) predicts outcome after cardiac arrest and induced hypothermia. Intensive Care Med 2006, 32:836-842.

14. Legriel S, Hilly-Ginoux J, Resche-Rigon M, Merceron S, Pinoteau J, HenryLagarrigue M, Bruneel F, Nguyen A, Guezennec P, Troché G, Richard O, Pico F, Bédos JP: Prognostic value of electrographic postanoxic status epilepticus in comatose cardiac-arrest survivors in the therapeutic hypothermia era. Resuscitation 2013, 84:343-350.

15. Rossetti AO, Oddo M, Liaudet $L$, Kaplan PW: Predictors of awakening from postanoxic status epilepticus after therapeutic hypothermia. Neurology 2009, 72:744-749.

\section{$10.1186 / \mathrm{cc} 13102$}

Cite this article as: Legriel: Electroencephalographic monitoring in comatose survivors of cardiac arrest. Critical Care 2013, 17:1010 